\title{
Data on the Atomic Form Factor: Computation and Survey
}

\author{
Ann T. Nelms and Irwin Oppenheim
}

\begin{abstract}
This paper presents the results of calculations of atomic form factors, based on tables of electron charge distributions computed from Hartree wave functions, for a wide range of atomic numbers. Computations of the form factors for five elements - carbon, oxygen, iron, arsenic, and mercury - are presented and a method of interpolation for other atoms is indicated. A survey of previous results is given and the relativistic theory of Rayleigh scattering is reviewed. Comparisons of the present results with previous computations and with some sparse experimental data are made.
\end{abstract}

\section{Introduction}

The atomic form factor is of interest in the calculation of Rayleigh scattering of radiation and coherent scattering of charged particles from atoms in the regions where relativistic effects can be neglected. The coherent scattering of radiation from an atom consists of Rayleigh scattering from the electrons, resonant electron scattering, nuclear scattering, and Delbrück scattering. When the frequency of the incident photon approaches a resonant frequency of the atom, large regions of anomalous dispersion occur in which the form factor calculations are not sufficient to describe the coherent scattering.

The cross section for Rayleigh scattering from the bound electrons of an atom with a deflection $\phi,{ }^{1}$ per steradian $d \Omega$, is

$$
d \sigma=\frac{r_{0}^{2}}{2}\left(1+\cos ^{2} \phi\right)|f(\vec{q})|^{2} d \Omega
$$

where $r_{0}=e^{2} / m c^{2}=2.82 \times 10^{-13} \mathrm{~cm}$ is the classical electron radius, $f(\vec{q})$ is the atomic form factor, $\vec{q}$ is the change of momentum of the photon, and $|\vec{q}|=(h \nu / c) 2 \sin (\phi / 2)$, where $\nu$ is the frequency of the incident photon. Terms of order $v / c$, where $v$ is the initial velocity of the bound electron, are neglected in the derivation of (1).

The cross section for the coherent scattering of charged particles with a deflection $\phi$, per steradian $d \Omega$, is, in Born approximation:

$$
d \sigma=\left|\frac{\epsilon e}{2 M v^{2}}[Z-f \overrightarrow{(q)}] \operatorname{cosec}^{2} \phi / 2\right|^{2} d \Omega
$$

where $\epsilon$ is the charge of the scattered particle, $M$ is its mass, and $v$ its velocity. $Z$ is the atomic number of the atom from which the particle is scattered. Equation (2) is a good approximation for all angles if the kinetic energy of the incident particle is large and $Z$ is not very large. For lower kinetic energies, eq (2) is valid for small-angle scattering.

\footnotetext{
I In more usual notation $\phi=2 \theta$, where $\theta$ is the Bragg angle.
}

The form factor for an atom of atomic number $Z$ is defined as the matrix element:

$$
f(\vec{q})=\left(0\left|\sum_{j=1}^{Z} e^{\overrightarrow{i q} \cdot \overrightarrow{r_{j} / \hbar}}\right| 0\right)
$$

where 0 denotes the ground state of the atom, and $\vec{r}_{j}$ is the vector distance of the $j$ th electron from the nucleus. For a spherically symmetric atom

$$
f(\vec{q})=\int_{0}^{\infty} \rho(r) \frac{\sin k r}{k r} d r
$$

where $k=4 \pi(\nu / c) \sin (\phi / 2)=q / \hbar$

and $\rho(r)$ is the charge distribution of electrons.

The basic data for the form-factor calculations of this paper were the effective charge distributions in atoms recently tabulated by Freeman and others. The effective charge, $Z_{p}(r)$, may be defined in terms of the electron charge distribution

$$
\rho(r)=(1 / 4 \pi r) \frac{d^{2} Z_{p}(r)}{d r^{2}} .
$$

Because of the oscillatory nature of the integrand in eq (4), the calculation of the form factor must be done in a careful manner.

\section{Historical Survey}

The initial attempt to evaluate $\rho(r)$ numerically was made by Hartree [1], ${ }^{2}$ using the Bohr model of the atom. Atomic fields for a few elements were obtained from a numerical analysis of optical and $\mathrm{X}$-ray spectra from which core orbits were calculated. The results were generalized so that the form factor for an arbitrary ion could be calculated. Important data for the analysis of crystal structures were obtained in this manner, but the calculated form factors were, in general, larger than the observed values.

2 Figures in brackets indicate the literature references at the end of this paper. 
The advent of the wave mechanical theory of scattering by Wentzel and Waller [2] confirmed the general formulations (1) and (2) of the scattering process. Charge distributions were now to be determined from solutions of the appropriate Schrödinger equations. An exact solution is obtainable for the hydrogen atom but not for many-electron atoms. For a one-electron system

$$
\rho(r)=4 \pi r^{2} \psi^{*}(r) \psi(r),
$$

where $\psi(r)$ is the Schrödinger wave function. Some of the approximations used for many-electron atoms will be discussed below.

\subsection{Pauling and Sherman Model}

Pauling and Sherman [3] obtained form factors for all atoms and some ions from hydrogen-like eigenfunctions with screening constants characteristic of each subshell. Very good results for smallangle scattering in all materials and for intermediate angles in low- $Z$ materials are obtained. The method tends to overemphasize the shell structure of atoms, and one would expect more accurate results to lie between the Pauling-Sherman curves and the Fermi-Thomas curves.

\subsection{Hartree Self-Consistent Field Model}

The most accurate extensive computations of wave functions of many-electron atoms are based on Hartree's [4] self-consistent field method. This is an independent particle model in which each electron is assumed to be in the field of the nucleus and an average field due to the other electrons. Thus the charge distribution can be considered as being a sum of one-electron charge distributions

$$
\rho(r)=\sum_{j=1}^{Z} \rho_{j}(r)=4 \pi r^{2} \sum_{j=1}^{Z} \psi_{j}^{*}(r) \psi_{j}(r),
$$

where $\psi_{j}(r)$ is the wave function for the $j$ th electron. This treatment is applicable to ions, as well as atoms. Fock [5] has generalized the Hartree scheme by including the effects of exchange, but few computations of Hartree-Fock wave functions are available. Slater [6] has shown that the Hartree and Hartree-Fock wave functions arise out of variational treatments in which the wave function of the many-electron atom is assumed to be a product of individual electron wave functions.

James and Brindley [7] have utilized Hartree wave functions in the calculation of atomic form factors. They tabulate results for $1 \leq Z \leq 37$ and $0 \leq(\sin \phi / 2) / \lambda \leq 1.1$, using an interpolation method for elements not given by Hartree. Their results are presented in tables of form factors for subshells of electrons versus $[1 /(Z-s)][(\sin \phi / 2) / \lambda]$, where $s$ is a screening parameter determined empirically as a function of $Z$ and the subshell of electrons considered. The atomic form factor is obtained by summing the form factors for the various groups of electrons. The method provides a suitable means of interpolation. The calculation of the form factor involves the numerical integration of a product of an oscillating function and the charge distribution for each subshell. Computational errors are inherent in this procedure and when the results are summed to give atomic form factors appreciable errors may occur.

\subsection{Fermi-Thomas Model}

Fermi [8] and Thomas [9] treated the electronic cloud of an atom by Fermi-Dirac statistics and obtained a spherically symmetric electron atmosphere having a density

$$
\rho=\frac{Z}{4 \pi \mu^{3}}\left(\frac{\phi(X)}{X}\right)^{3 / 2}
$$

where $\mu=0.47 \times 10^{-8} \times Z^{-1 / 3} \mathrm{~cm}, X=r / \mu$, and $\phi(X) / X=\left(\mu / Z_{e}\right) V(r)$, where

$$
V(r)=\frac{Z e}{r}-e \int \frac{\rho\left(\overrightarrow{r^{\prime}}\right)}{\left|\vec{r}-\overrightarrow{r^{\prime}}\right|} d^{3} \overrightarrow{r^{\prime}}
$$

is the potential at $r$ due to the nuclear charge $Z$ and the electron charge distribution $\rho(r)$.

Form-factor calculations have been made as a function of the universal variable

$$
u=4 \pi \mu \sin (\phi / 2) / \lambda,
$$

where $\lambda$ is the wavelength of the incident radiation. The calculations have been applied to neutral atoms and positive ions and give smooth electron distributions that average the effects from each electron. This approximation is fairly good for high- $Z$ materials over most of the electron distribution but leads to large discrepancies with experiment at small and large angle scattering. The inherent limitations of the statistical method are the following:

(a) The effects of atomic shell structure are smoothed out.

(b) A poor distribution is obtained where the potential is small (at large distances from the nucleus) and where it changes rapidly (close to the nucleus).

A discussion of the inadequacies of this model for incoherent scattering is given by White [10].

Gaspar [11] has noted that reduced atomic form factors determined by the self-consistent field method, can be regarded as almost universal functions of the Fermi-Thomas variable

$$
u=\frac{0.8853}{Z^{1 / 3}} a_{0} k=0.8853 U,
$$

where $k=4 \pi(\sin \phi / 2) / \lambda=q / \hbar$, and $a_{0}$ is the Bohr radius. As is evident from figures 1 and 2 , this is true only for values of $U<3$. There are appreciable differences at higher values of $U$ due to the shell structure of the atoms. 


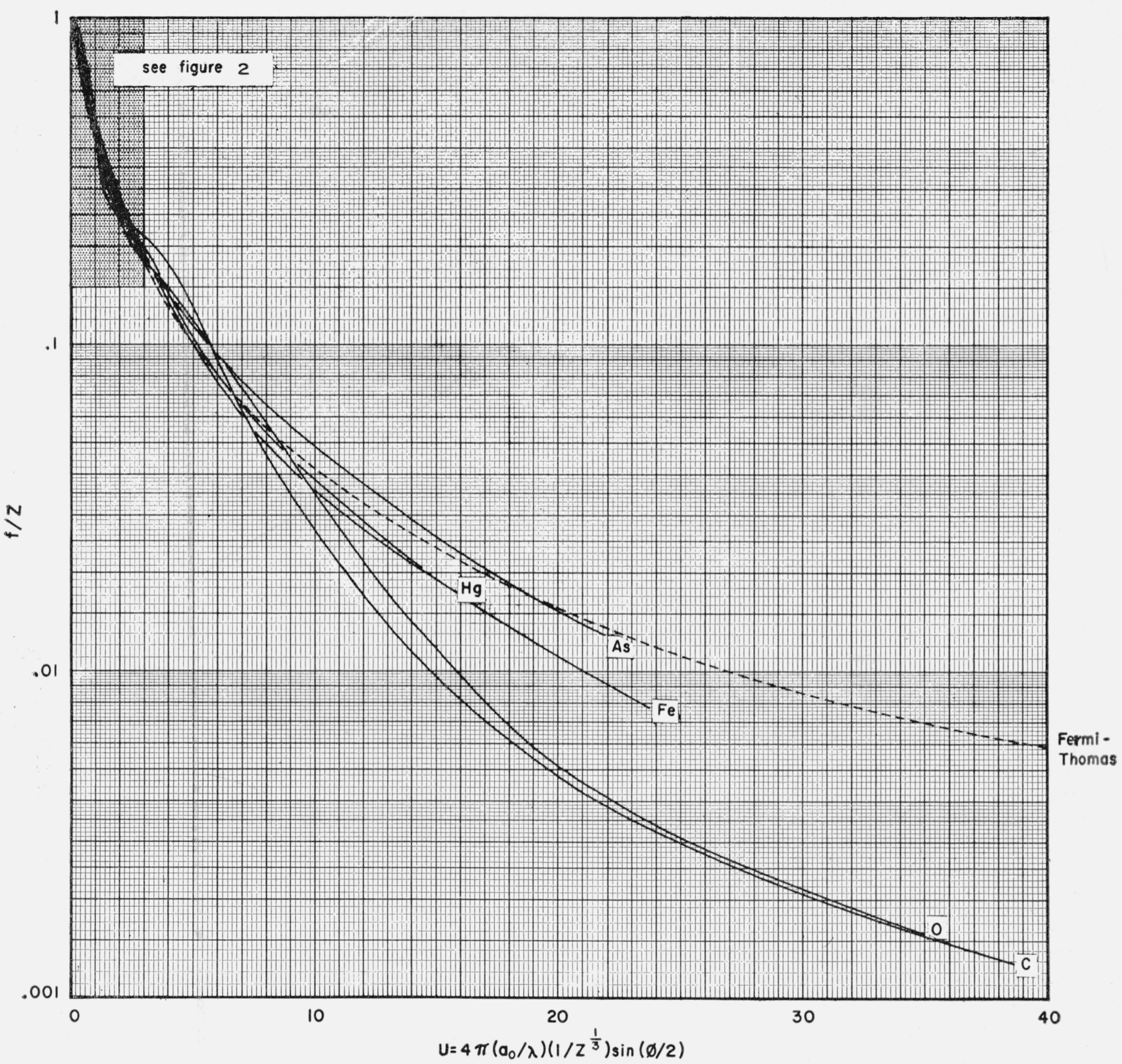

Figure 1. Comprehensive plot of form factor values per electron, $a_{0}=0.528 \times 10^{-8} \mathrm{~cm}, \lambda=$ wavelength. 


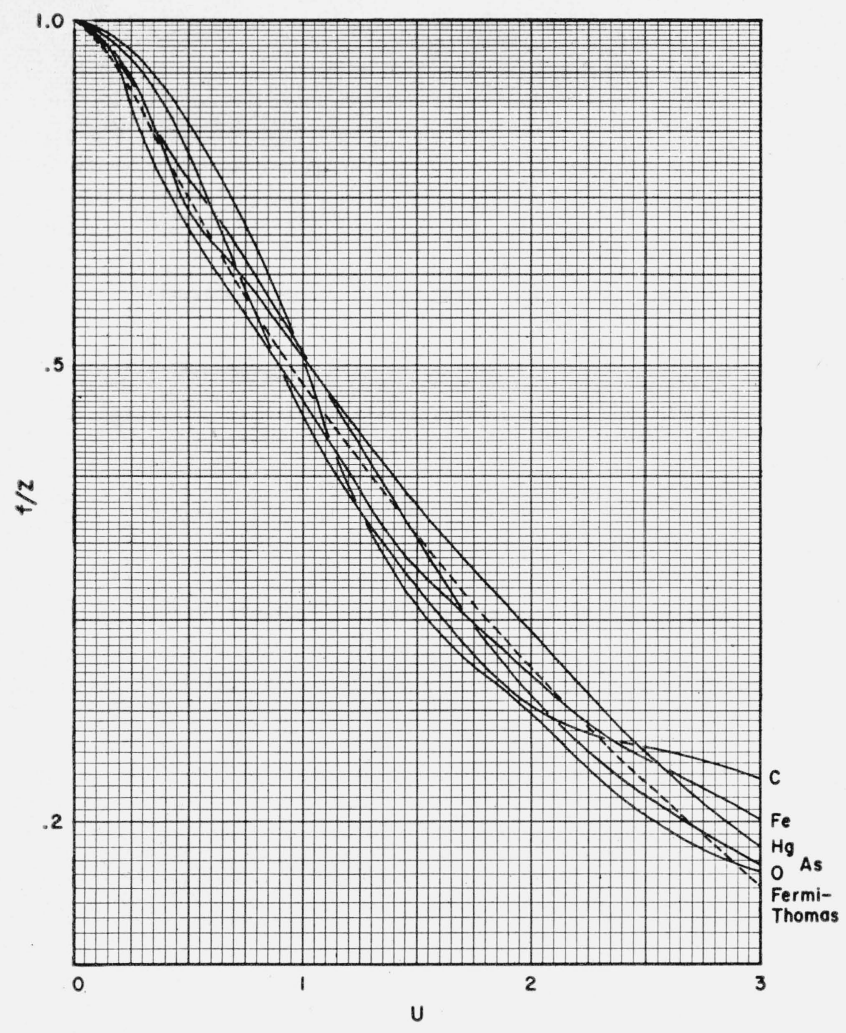

Figure 2. Form factor per electron for $U \leq 8$.

\section{Relativistic Effects}

Relativistic effects are of importance for heavy atoms where the inner electrons have relativistic velocities and for scatterings in which the momentum change of the photon is of the order of or greater than mc. Calculations in this report have been carried up to momentum changes less than or equal to $m c / 2$.

Franz [12] has shown that the relativistic expression for scattering of a high-energy photon by a bound electron reduces to the form-factor calculation when $q \ll m c$ and the velocity of the electron is nonrelativistic, i. e., $(v / c)^{2} \sim(\alpha Z / n)^{2} \ll 1$, where $\alpha$ is the fine-structure constant, and $n$ is the principal quantum number of the electron. ${ }^{3}$ His method of calculation utilizes plane waves for the electron wave functions in the intermediate states. (The Dirac perturbation theory describes scattering as a twostep process, absorption followed by reemission or emission followed by absorption; thus one must consider the state of the electron between the two steps.) The effect of electron binding in the intermediate state is thus neglected. For his computation of the form factor, Franz uses a Fermi-Thomas distribution.

\footnotetext{
${ }^{3}$ Note that Franz's equation [12], which is valid for scattering of high energy photons only, is in error by a factor of 2 due to a mistake in transforming variables of integration over the angle of scattering. Thus we have for the total cross section $\sigma_{\mathrm{coh}}=2 \sigma_{\mathrm{Franz}}$.
}

Levinger [13] has considered corrections to the form factor calculation in the near relativistic region but has neglected the effect of binding in the intermediate states. Bethe [14] has confirmed Franz's result and has shown that for values of $q$ larger than the characteristic momentum of a $K$ electron $m c Z / 137$, Dirac wave functions should be used for the computation for $K$ electrons. Rohrlich and Rosenzweig [15] have extended Bethe's results to a treatment of $L$ electrons.

Brown and Woodward [16] have investigated the effect of binding in the intermediate states and find further corrections to the form-factor calculations of the same order of magnitude as Levinger's. An extensive project by Brown et al at Birmingham in the exact calculation of Rayleigh cross sections for selected atoms and energies is now under way. Results for the scattering of $0.32 m c^{2}$ gamma rays from the $K$ electrons of mercury [17] indicate that the form factor results are a reasonably valid description of the dispersive scattering but not of the total scattering which includes contributions from absorptive scattering. Under the conditions of the calculation, the absorptive scattering is approximately $1 / 6$ of the dispersive contribution.

\section{Form-Factor Calculations}

The form-factor calculations of this report are based on the published numerical data of Freeman [18] and others [19], who calculated the effective charge distribution in atoms, $Z_{p}(r)$, from the Hartree self-consistent field model. ${ }^{4}$ Hartree-Fock data were not used because they were unavailable in quantity. It is well to note that, although the radial charge distributions of the Hartree and Hartree-Fock models differ considerably, the corresponding form factors may not. Brindley and Ridley [20] have computed form factors for $\mathrm{K}^{+}, \mathrm{Cl}^{-}$, and $\mathrm{Cu}^{+}$, using both Hartree and Hartree-Fock wave functions. Their computations, which cover the range from $k=0$ to $k=2 \pi$, indicate that the form factors with exchange are higher than those without exchange. The maximum difference in the form factors is of the order of 3 percent in this small-angle region. A trial calculation in the present work for $\mathrm{Cu}^{+}$at $k=10$ indicates a difference in the form factors of 0.6 percent between the Hartree and Hartree-Fock results.

The effective charge distribution $Z_{p}(r)$ is defined by the relation (5)

$$
\rho(r)=\left(\frac{1}{4 \pi r}\right) \frac{d^{2} Z_{p}(r)}{d r^{2}},
$$

where $\rho(r)$ is the charge density of electrons in the atom. Equation (4) may be rewritten, by means of partial integration to give

$$
f(k)=Z-\int_{0}^{\infty} Z_{p}(r) \sin (k r) d(k r),
$$

${ }^{4}$ An upper limit of $k=70(q=m c / 2)$ was established for this calculation so that relativistic effects could be neglected without serious error. 
where $k=q / \hbar$. In the tables of [18], $r$ is given in units of the Bohr radius, and $k$ must be expressed in the reciprocal units

$$
k a_{0}=(4 \pi / \lambda) \sin (\phi / 2) a_{0}=6.635 \sin (\phi / 2) / \lambda .
$$

The numerical data, $Z_{p}(r)$, for each atom were fitted empirically by an expansion of the form

$$
e^{-\alpha r} \sum_{i=0}^{n} a_{i} r^{i}
$$

Functions of this form were chosen so that direct comparison could be made with the effective charge of hydrogen type atoms

$$
Z_{p}(r)=e^{-2 r}(Z+r Z) .
$$

The fitting procedure for nonequidistant values of $r$ is described in detail in [21]. The $Z_{p}(r)$ data were fitted in several intervals separately. This was done to obtain a higher degree of accuracy without using expansions involving large numbers of terms. In this way, six-term expansions $(n=5)$, at most, were necessary to fit the data. Values of the coefficients for each interval for the five atoms considered $-\mathrm{C}$, $\mathrm{O}, \mathrm{Fe}, \mathrm{As}, \mathrm{Hg}$ - are listed in table 1. It is, perhaps, only in the first interval that one can make a significant comparison with the effective charge of hydrogen-type atoms. The accuracy of the tabulated $Z_{p}(r)$ and of the fitting is noted also in table 1 . In all cases effort was expended to make the fit as accurate as the data.

Substitution of (11) into (10) results in

$$
\begin{aligned}
& \frac{f(k)}{Z}=1-(k / Z) \sum_{m} \sum_{i} a_{i}^{(m)}(-1)^{i} \\
& \frac{\partial^{i}}{\partial \alpha^{i}} \int_{r_{m-1}}^{r_{m}} e^{-\alpha^{(m)} \tau} \sin (k r) d r,
\end{aligned}
$$

where the sum over $m$ denotes a sum over the various intervals for which $Z_{p}(r)$ was fitted, $r_{0}=0$ and $r_{m_{\max }}=\infty$, and the superscript $(m)$ on the coefficients $a_{i}$ and $\alpha$ denote the values for the appropriate intervals. An analytic integration was obtained in this way for $0.5 \leq k \leq 20$ (fig. 3 and table 2). The maximum error introduced by the fitting procedure in the calculation can be easily estimated by assuming that the deviation of the fit from the tabulated value is in phase with the oscillating $\sin (k r)$. An error of 3 percent is estimated in this way. However, it is improbable that the actual error is of this magnitude. A more realistic value would be of the order of 1 percent. Although this

\begin{tabular}{|c|c|c|c|c|c|}
\hline \multirow{2}{*}{$k$} & \multicolumn{5}{|c|}{$Z$} \\
\hline & 6 & 8 & 26 & 33 & 80 \\
\hline $\begin{array}{r}0.0 \\
0.5 \\
1.0 \\
2.0 \\
3.0 \\
4.0 \\
5.0 \\
6.0 \\
8.0 \\
10.0 \\
20.0\end{array}$ & $\begin{array}{l}1.0 \\
0.912 \\
.721 \\
.415 \\
.296 \\
.251 \\
.226 \\
.204 \\
.160 \\
.104 \\
.0217\end{array}$ & $\begin{array}{l}1.0 \\
0.944 \\
.809 \\
.508 \\
.308 \\
.249 \\
.202 \\
.181 \\
.150 \\
.118 \\
.0386\end{array}$ & $\begin{array}{l}1.0 \\
0.942 \\
.759 \\
.582 \\
.461 \\
.360 \\
.306 \\
.266 \\
.217 \\
.182 \\
.0785\end{array}$ & $\begin{array}{l}1.0 \\
0.944 \\
.842 \\
.675 \\
.540 \\
.426 \\
.338 \\
.277 \\
.211 \\
.178 \\
.0890\end{array}$ & $\begin{array}{l}1.0 \\
0.960 \\
.900 \\
.700 \\
.616 \\
.530 \\
.460 \\
.400 \\
.314 \\
.250 \\
.109\end{array}$ \\
\hline
\end{tabular}
calculation procedure is straightforward, the length of the calculation and inability to make general checks make it a tedious operation. ${ }^{5}$

TABLE 2. Form factor, $f / Z$, tabulated from eq (13)

The behavior of the form factor for small $k, k<$ 0.5 , was determined by expanding $\sin k r$ in powers of $k r$ :

$$
f(k)=Z+\sum_{n=1}^{\infty} \frac{(-k)^{2 n}}{(2 n-1) !} \int_{0}^{\infty} Z_{p}(r) r^{2 n-1} d r .
$$

\begin{tabular}{|c|c|c|c|c|c|c|c|c|c|c|}
\hline 7 & Interval, $m$ & $\alpha$ & $a_{\theta}$ & $a_{1}$ & $a_{2}$ & $a_{3}$ & $a_{4}$ & $a_{5}$ & $\begin{array}{l}\text { Maxi- } \\
\text { mum } \\
\text { error of } \\
\text { calcula- } \\
\text { tions }\end{array}$ & $\begin{array}{c}\text { Maxi- } \\
\text { mum } \\
\text { error in } \\
\text { reference } \\
{[18]}\end{array}$ \\
\hline C.... & 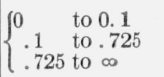 & $\begin{array}{l}2.475 \\
2.5\end{array}$ & $\begin{array}{l}6 \\
6.04511 \\
4.84325\end{array}$ & $\begin{array}{r}-4.98448 \\
1.90496\end{array}$ & $\begin{array}{r}18.66594 \\
-1.23017\end{array}$ & $\begin{array}{c}-11.06320 \\
0.158\end{array}$ & & & $\begin{array}{r}0.01 \\
.01 \\
.01\end{array}$ & 0.01 \\
\hline $0 \ldots$ & $\begin{cases}0 & \text { to } 0.1 \\
.1 & \text { to } .8 \\
.8 & \text { to } \infty\end{cases}$ & $\begin{array}{l}2.77 \\
2 \\
1.91\end{array}$ & $\begin{array}{l}8 \\
7.673 \\
7.764\end{array}$ & $\begin{array}{r}-3.622 \\
0.917\end{array}$ & $\begin{array}{l}13.42 \\
-1.884\end{array}$ & $\begin{array}{c}-10.34 \\
0.395\end{array}$ & & & $\begin{array}{l}.02 \\
.01 \\
.003\end{array}$ & 0.02 \\
\hline $\mathrm{Fe}_{-}$ & 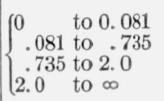 & $\begin{array}{l}3.7283 \\
2.35866 \\
1.3966 \\
1.07624\end{array}$ & $\begin{array}{l}26 \\
24.68559 \\
24.11502 \\
5.45076\end{array}$ & $\begin{array}{r}-20.3322 \\
-15.43486 \\
-24.18032 \\
1.28879\end{array}$ & $\begin{array}{c}256.0535 \\
-62.94642 \\
14.23867 \\
-0.07209\end{array}$ & $\begin{array}{l}-61.5426 \\
548.52436 \\
-2.27092 \\
-0.00950\end{array}$ & -1032.86606 & 610.402956 & $\begin{array}{l}.02 \\
.11 \\
.0001\end{array}$ & 0.03 \\
\hline As .... & $\begin{cases}0 & \text { to } 0.1 \\
.1 & \text { to } .7 \\
.7 & \text { to } 1.8 \\
1.8 & \text { to } \infty\end{cases}$ & $\begin{array}{l}4.5 \\
2.6 \\
1.27713 \\
1.14162\end{array}$ & $\begin{array}{l}33 \\
34.28291 \\
\text { 39. } 90909 \\
9.79098\end{array}$ & $\begin{array}{l}-20.55955 \\
-89.42302 \\
-94.09721 \\
-0.14061\end{array}$ & $\begin{array}{r}671.12959 \\
464.62137 \\
122.21415 \\
0.24590\end{array}$ & $\begin{array}{r}-2603.64918 \\
-1124.45247 \\
-79.41765 \\
-0.038708\end{array}$ & $\begin{array}{r}192.72860 \\
26.37782\end{array}$ & $\begin{array}{r}-551.527777 \\
-3.56417\end{array}$ & $\begin{array}{l}.01 \\
.03 \\
.002 \\
.003\end{array}$ & 0.03 \\
\hline $\mathrm{Hg}_{-}$. & $\left\{\begin{array}{l}0 \text { to } 0.106 \\
.106 \text { to } .478 \\
478 \text { to } 1.96 \\
1.96 \text { to } \infty\end{array}\right.$ & $\begin{array}{l}5.14277 \\
3.21425 \\
1.68832 \\
1.09654\end{array}$ & $\begin{array}{r}80 \\
45.99829 \\
-14.19283 \\
31.56632\end{array}$ & $\begin{array}{l}-59.11228 \\
637.7033 \\
242.09358 \\
-23.61074\end{array}$ & $\begin{array}{r}-2524.22231 \\
-6844.95947 \\
-469.93545 \\
9.58349\end{array}$ & $\begin{array}{r}124272.83758 \\
31986.00973 \\
420.91024 \\
-1.72085\end{array}$ & $\begin{array}{r}-1566263.92891 \\
-67636.26526 \\
-178.44611 \\
0.13744\end{array}$ & $\begin{array}{r}6462387.83717 \\
52950.76587 \\
29.20347 \\
-0.00386\end{array}$ & $\begin{array}{l}.03 \\
.01 \\
.04\end{array}$ & 0.025 \\
\hline
\end{tabular}

${ }^{5}$ Note that a graphical procedure would not have been suitable in this range because of the oscillating nature of the integrand.

TABLE 1. Coefficients in the expansion $Z_{p}^{(m)}(r)=e^{-a^{(m)}} \sum_{i} a_{i}{ }^{(m)} r^{i}$ 


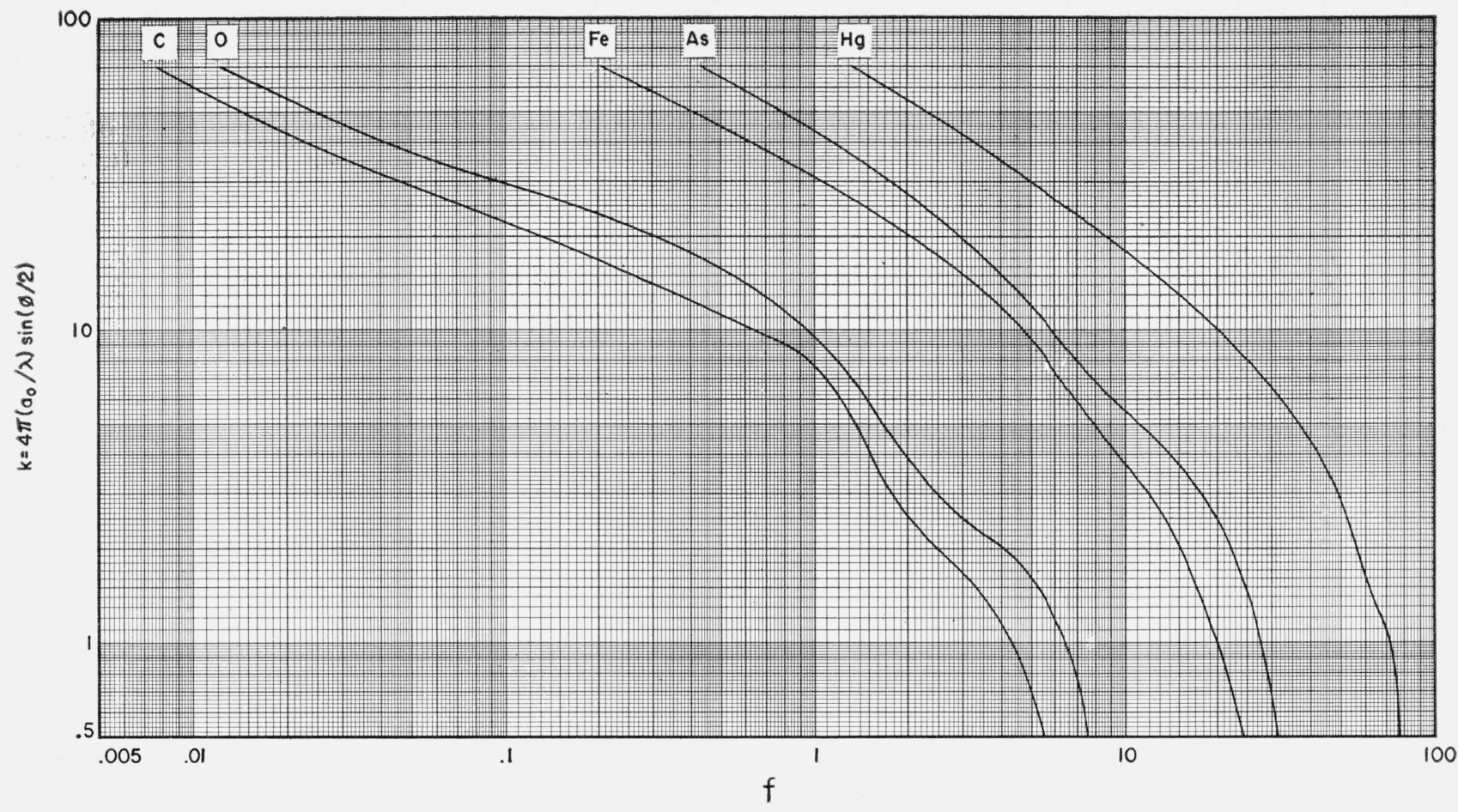

FiguRe 3. Form factor in the range $0.5 \leq k \leq 70$.

At the origin, one obtains:

$$
f(0)=Z \text { and }\left(\frac{d f(k)}{d k}\right)_{k=0}=0 .
$$

Near the origin:

$$
f(k)=Z+k^{2} \int_{0}^{\infty} Z_{p}(r) r d r .
$$

The integral in eq (15) was evaluated graphically with the tabulated $Z_{p}(r)$ (see fig. 4 ).

It is of interest to note the connection of the form factor for small-angle scattering with the expression for the gram-atomic diamagnetic susceptibilities. The expression for the susceptibility, $\chi_{\mathrm{d} 1 \mathrm{a}}$, is

$$
\chi_{\text {dia }}=-\left(8 \times 10^{-7}\right) a_{0}^{-2}\left(\left.0\left|\sum_{j}\right| r_{j}\right|^{2} \mid 0\right),
$$

where $a_{0}$ is the Bohr radius. For spherically symmetric ators, we have

$$
\left(\left.0\left|\sum_{j}\right| r_{j}\right|^{2} \mid 0\right)=6 \int_{0}^{\infty} Z_{p}(r) r d r
$$

and from (15)

$$
f(k)=Z+\frac{k^{2}}{6}\left(1.25 \times 10^{6}\right)\left(-\chi_{\mathrm{dia}}\right) a_{0}^{2} .
$$

The integral in (17) involves a precise knowledge of the effective charge, or the wave function at large radii and is therefore strongly dependent on the chemical binding of the atom. Since the tabulated

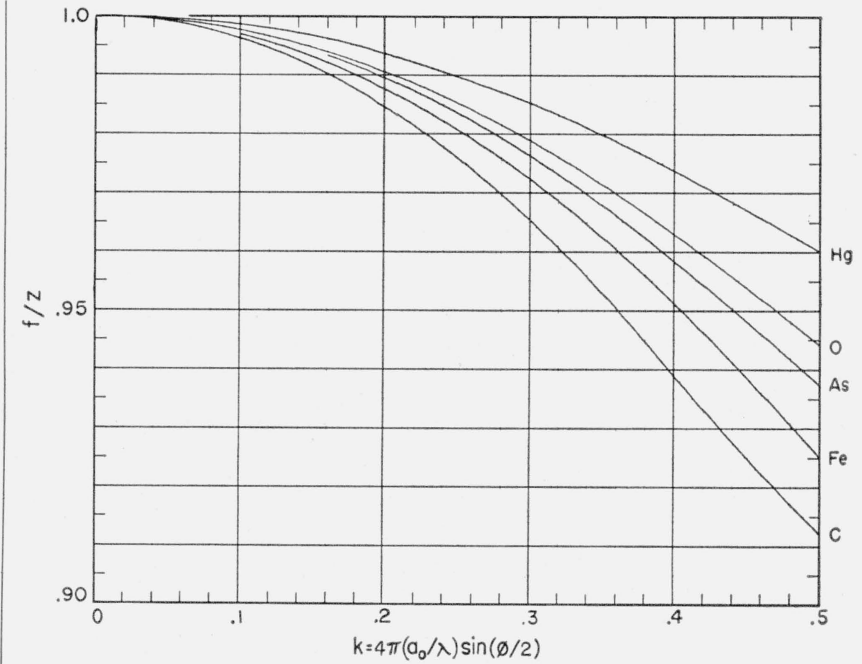

FIGURE 4. Small-angle approximation of the form factor per electron.

$Z_{p}(r)$ are for free atoms, and since the experimental $\chi_{\text {dia }}$, as well as most data on the form factor from $\mathrm{X}$-ray and electron small-angle diffraction, is measured for atoms either bound in molecules or crystals, a comparison between the computation and experiment is not quite direct. Brindley and Hoare [22] have discussed the difficulty in evaluating susceptibilities from experimental data. Nevertheless, the diamagnetic susceptibilities computed from the 
values of $Z_{p}$ used in this paper agree with the experimental values obtained for the atom in chemical combination to within 3 to 10 percent (see table 3 ).

TABLE 3. Gram-atomic susceptibility, $-\chi_{A} \times 10^{6}$

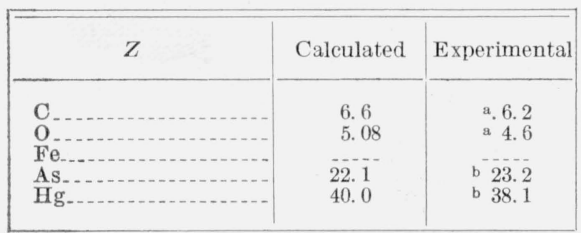

${ }^{a}$ E. C. Stoner, Magnetism (Methuen and Co. Ltd., 36 Essex Street, W. C., London, 1930).

b Handbook of Chemistry and Physies, 30th ed. (Chemical Rubber Publishing Co., Cleveland, Ohio).

For $k>20$, rough calculations were made because of the uncertainty of errors due to relativistic effects. For large $k$ a useful expansion of (10) is

$$
\begin{aligned}
& f(k)=-\frac{1}{2} \int_{0}^{\pi / k} G(r) \cos (k r) d r \\
&+\frac{1}{2} \int_{0}^{\infty} \sum_{1}^{\infty}\left(\frac{\pi}{k}\right)^{n} \frac{G^{(n)}(r)}{n !} \cos (k r) d r \\
&=-\frac{1}{2} \int_{0}^{\pi / k} G(r) \cos (k r) d r+\frac{\pi}{4 k} \int_{0}^{\pi / k} G^{(1)}(r) \cos (k r) d r \\
& \quad-\frac{1}{48}\left(\frac{\pi}{k}\right)^{3} \int_{0}^{\pi / k} G^{(3)}(r) \cos (k r) d r \\
& \quad+\frac{1}{8} \int_{0}^{\infty} \sum_{4}^{\infty}\left(\frac{\pi}{k}\right)^{n} \frac{G^{(n)}(r)}{n !} \cos (k r) d r
\end{aligned}
$$

where

$$
G(r)=\frac{1}{Z} \frac{d Z_{p}(r)}{d r} \text { and } G^{(n)}(r)=\frac{d^{n}}{d r^{n}} G(r)
$$

The coefficient of the $G^{(2)}$ integral is zero. The approximation used was

$$
\begin{array}{r}
f(k)=-\frac{1}{2} \int_{0}^{\pi / k} G(r) \cos (k r) d r+\frac{\pi}{4 k} \int_{0}^{\pi / k} G^{(1)}(r) \cos (k r) d r \\
-\frac{1}{48}\left(\frac{\pi}{k}\right)^{3} \int_{0}^{\pi / k} G^{(3)}(r) \cos (k r) d r . \quad(20)
\end{array}
$$

As is to be expected, only regions close to the nucleus contribute to the form factor for large momentum changes. These computations were compared with a hydrogen-like approximation, using Slater screening constants for low- $Z$ materials. For high $Z$ a comparison with a Thomas-Fermi calculation was made. The comparisons were informative for general trends only and could not be used as a test of accuracy.

An interpolation scheme is given in figure 5 over the range for which calculations were made, $0.5 \leq k \leq 20$. Tabulations of the form factor by James and Brindley, which are based on Hartree wave functions, were used as a guide in regions where we had little calculated data and as a general test of interpolation in other areas. The curves are plotted with 1-percent accuracy, and interpolation can vield results within 2 percent. A more direct test of the interpolation was made by comparing the form factor of $\mathrm{Cu}^{+}(k=10)$, obtained graphically from published data [23] of $Z_{p}(r),(f=5.26)$, with the interpolated value for $\mathrm{Cu}(f=5.23)$.

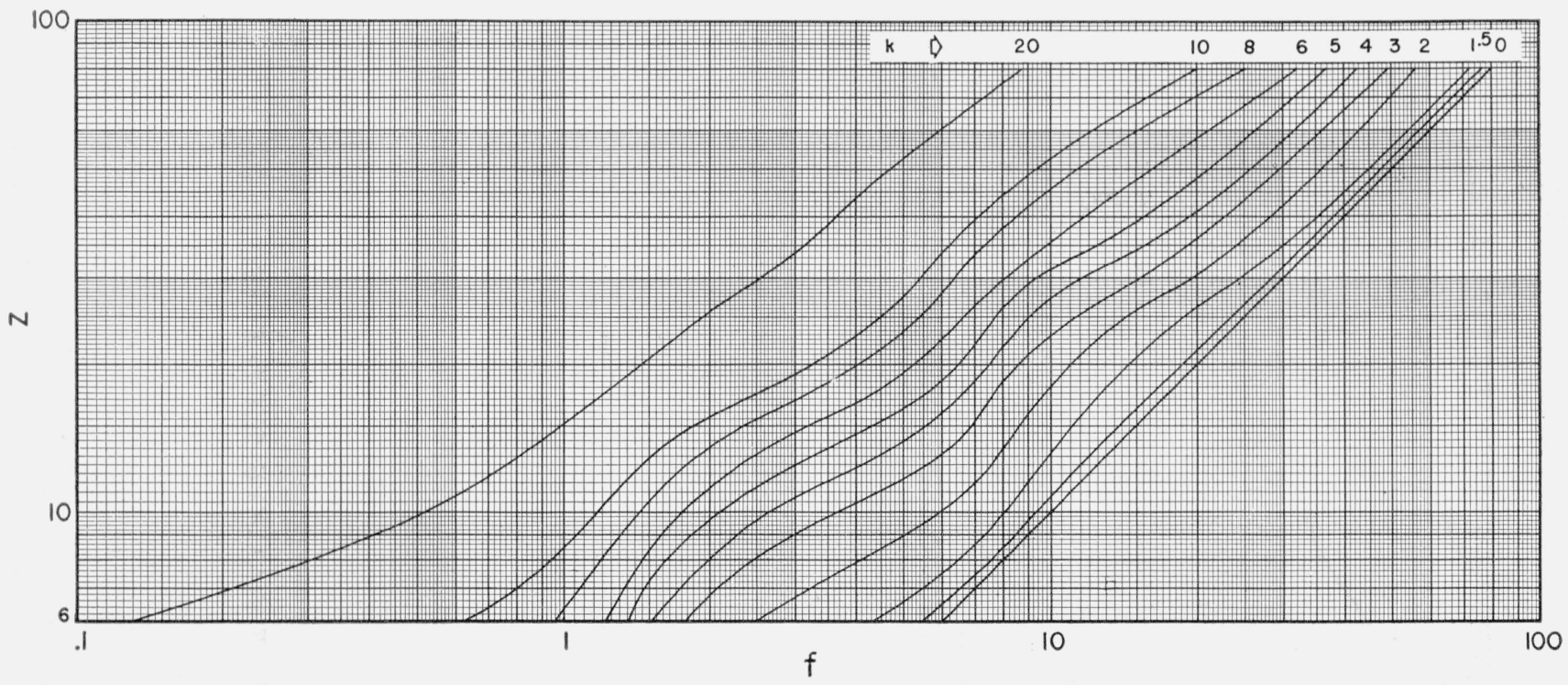

FiguRe 5. Form factor as a function of $Z$ in the range $0 \leq k \leq 20, k=4 \pi\left(a_{n} / \lambda\right) \sin (\phi / 2)$. 


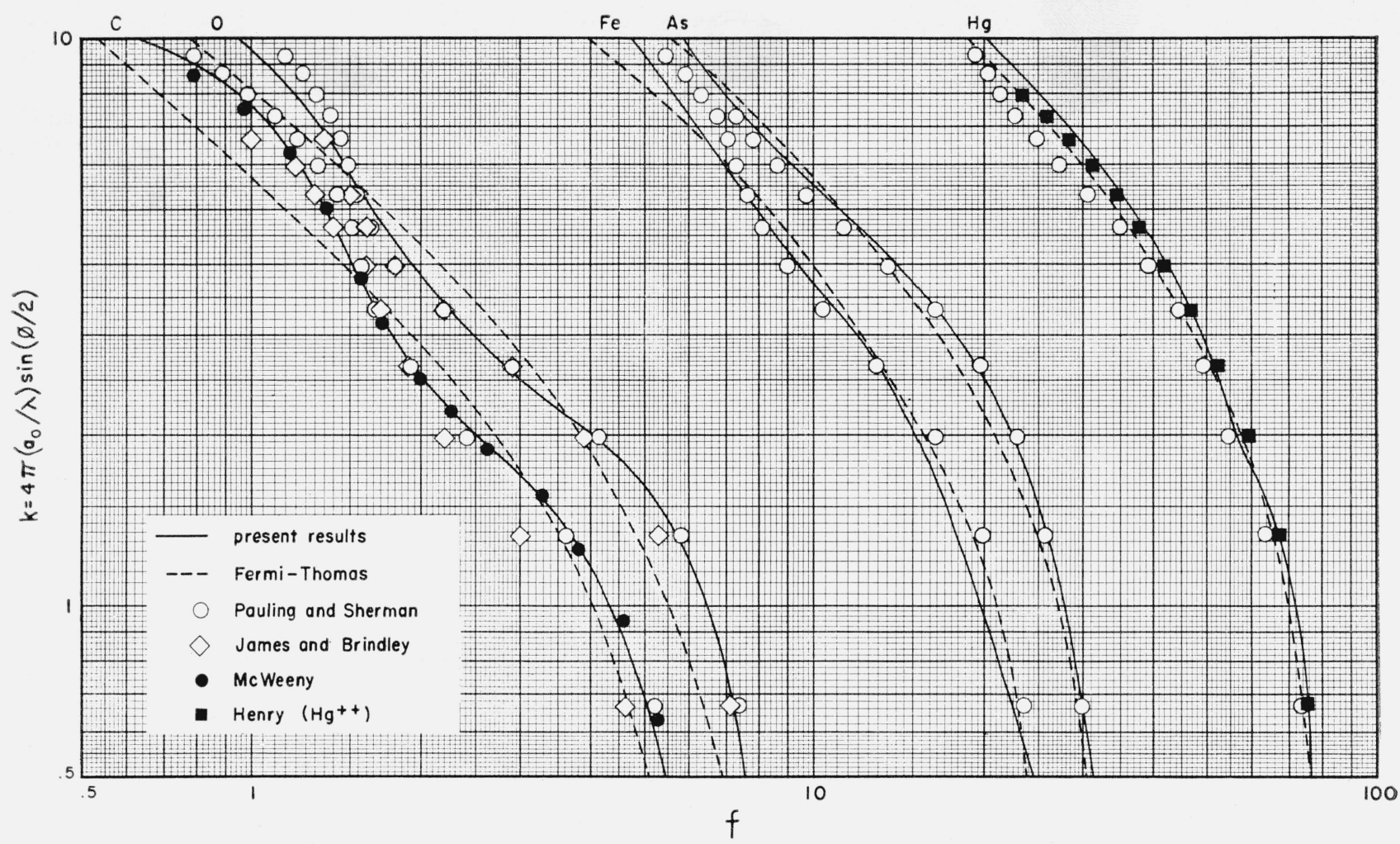

FiguRE 6. Comparison of calculated form factors from several models.

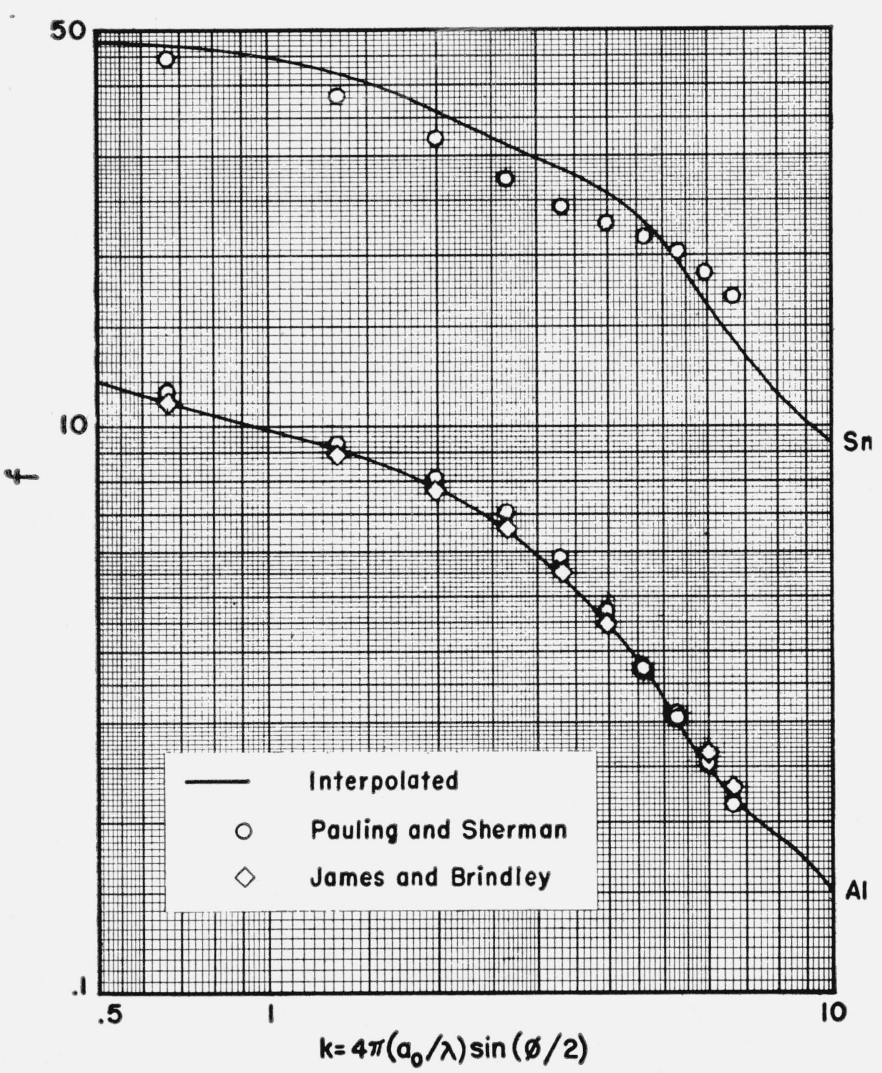

\section{Comparisons With Other Calculations}

Comparisons of the present results with the numerical results of the approximations discussed in section 3 are given in figure 6 for $\mathrm{C}, \mathrm{O}, \mathrm{Fe}, \mathrm{As}$, and $\mathrm{Hg}$. Figure 7 shows a comparison of the results for $\mathrm{Sn}$ and $\mathrm{Al}$ obtained from the interpolation curves, figure 5, with the calculations of section 3 .

As is to be expected, the data of Pauling and Sherman agree best for low $Z$ with the present more exact calculations. In general, the oscillations in the Pauling-Sherman form-factor curves due to shell structure are larger than in the more exact. calculations.

The calculated curves oscillate about the FermiThomas curves, which average out the shell structure.

Comparison with the results of James and Brindley shows agreement over most of the range. The present form-factor computations are higher than the James and Brindley calculations for small and moderately large values of $k$, especially for $C$ in the range $0.655<k<2$. The results reported here show close agreement with the extensive computations by McWeeny [24] and others on the form factor of $\mathscr{C}$.

The present authors judge the results of this paper to be more accurate than those of James and Brindley

Figure 7. Comparison of interpolated form factors with results from other calculations. 
because the present computations utilize more recent charge-distribution data [18] and also avoid the errors inherent in the sum over subshell contributions.

The ratio of the cross sections for coherent and incoherent scattering should be a smooth function of $Z$. The value of this ratio for $\mathrm{C}$ obtained from McWeeny's calculations and from our own fits in well with the values for other light elements whereas the values obtained from James and Brindley's calculations do not. This anomaly of James and Brindley's data for $\mathrm{C}$ was apparent even in an analysis of total absorption coefficients (scattering and photoelectric) and contributed to a stimulation of the present study.

For high $Z$ materials, $Z>26$, comparisons are made with the Fermi-Thomas and Pauling and Sherman calculations. As was expected, the more exact calculations approach the Thomas-Fermi results as $Z$ increases. Even for $\mathrm{Hg}$, however, some effect of shell structure is apparent. A recent computation of the form factors for $\mathrm{Hg}^{++}$by Henry [25] involving a numerical integration of Hartree wave functions is plotted for comparison in figure 6 .

In figure 7 , interpolated form factors are compared with those of Pauling and Sherman for Sn and with those of Pauling and Sherman and of James and Brindley for $\mathrm{Al}$. There are deviations of approximately 17 percent from the data of Pauling and Sherman for $k=2.64$.

\section{Comparison With Experiment}

In the range for which our results are valid, i. e., nonrelativistic momentum changes, and in which there are significant differences in the results of the various form factor calculation, $U>3$, there are very few experimental data with which the calculations may be compared. The early X-ray data give information only for small momentum changes, $U<3$ in figures 1 and 2 , where the Hartree and Pauling-Sherman approximations approach the "universal" Fermi-Thomas form factor calculation very closely. Recent experiments by Wilson [26] and Mann [27] were not used because the momentum changes were larger than $m c / 2$, the limit established for the present calculation.

Comparisons are made with the experiments of Storruste [28] with 0.411-Mev incident photons on $\mathrm{Pb}$ and $\mathrm{Cu}$ (fig. 8). The experimental error is indicated by the size of the experimental points. Satisfactory agreement is obtained throughout the range though the experimental points for momentum changes greater than $m c / 6$ lie above the theoretical curve for $\mathrm{Pb}$.

The form factors that have been computed here are for Rayleigh scattering from the electrons of free, spherically symmetric atoms. McWeeny [24] has made extensive studies of the effects of asymmetry on the form factors, particularly for C. Using analytic wave functions of the Duncanson and Coulson type [29], he finds effects of the order of 3 percent on low-and medium-angle-scattering factors.

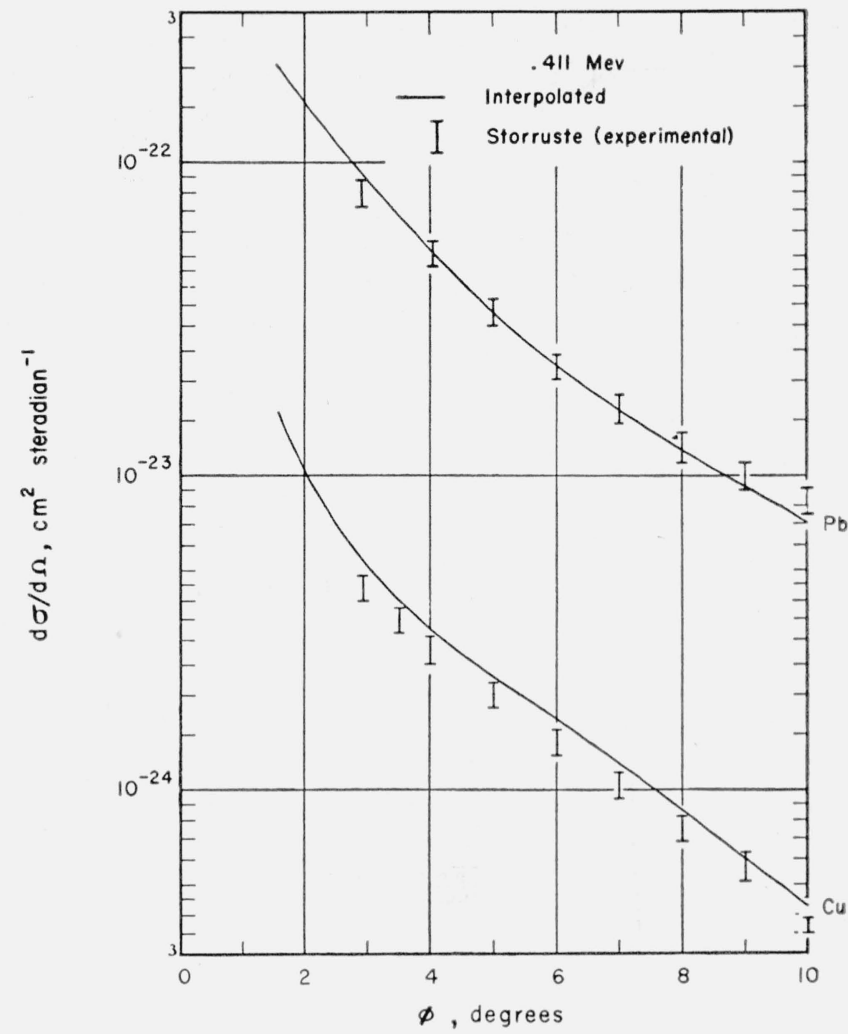

Figure 8. Comparison of cross section based on interpolated form factors with experimental data.

Experimental determinations of coherent scattering are almost always made with atoms bound either in molecules or in solids. Interatomic binding will distort the electron cloud producing a modification in electron charge distribution and therefore in the form factor. This binding will affect the small angle scattering of photons whose wavelengths are of the order of atomic dimensions, $(1$ to $10 \mathrm{~A})$. For higher energy photon scattering only the electron distribution near the nucleus, which is hardly influenced by interatomic binding, is of importance. Brill [30] has discussed the effect of interatomic binding, particularly in crystals, and finds effects of the order of 4 to 5 percent for $[\sin (\phi / 2)] / \lambda \sim 0.5$. McWeeny has found no significant difference between form factors computed for the valence states of free atoms and the form factors for these atoms bound in crystals.

Furthermore, as mentioned above, coherent scattering from atoms consists of Rayleigh scattering, resonant electron scattering, Delbrück scattering, and nuclear scattering. Resonant electron scattering produces large regions of anomalous dispersion when the frequency of the incident photons approaches a resonant frequency of the atom. Parratt [31] has shown that within a large frequency range, near and below the $K$ edge, e. g., $0.1 \mathrm{~A}<\lambda<100 \mathrm{~A}$ for 
$\mathrm{Cu}$, there is practically no region of normal dispersion. The form-factor calculation is not sufficient to obtain the coherent scattering in this region.

For a comparison with experimental results at higher frequencies, the cross sections for the Rayleigh, Delbrück, and nuclear processes must be known, as well as the phase relationships between the scatterings by each process. Rayleigh and nuclear scattering will constructively interfere up to several million electron volts while Delbrück scattering is out of phase with them in this range.

The inapplicability of the Born approximation (2) to the scattering of electrons by heavy atoms has been considered by Glauber et al. [32]. Tabulations of form factors corrected for deviations from the Born approximation have been given by Ibers and Hoerni [33].

The autbors thank U. Fano and G. R. White for many helpful discussions and I. Hornstein for aid in the computations.

\section{References}

[1] D. R. Hartree, Phil. Mag. 50, 289 (1925).

[2] (a) G. Wentzel, Z. Physik 43, 1 (1927); (b) I. Waller, Phil. Mag. 4, 1228 (1927); Nature 120, 155 (1927); Nature 15, 969 (1927); Z. Physik 51, 213 (1928).

[3] L. Pauling and J. Sherman, Z. Krist 81, 1 (1932).

[4] D. R. Hartree, Proc. Cambridge Phil. Soc. 24, 89, 111 (1928).

[5] V. Fock, Z. Physik 61, 126 (1930).

[6] J. C. Slater, Phys. Rev. 35, 210 (1930).

[7] R. W. James and G. W. Brindley, Phil. Mag. 12, 81 (1931).

[8] E. Fermi, Z. Physik 48, 73 (1928).
[9] L. H. Thomas, Proc. Cambridge Phil. Soc. 23, 542 (1926).

[10] G. R. White (unpublished NBS Report).

[11] R. Gaspar, Acta Phys. Acad. Sci. Hung. 3, No. 1, 59 (1953).

[12] W. Franz, Z. Physik 95, 652 (1935); 98, 314 (1936).

[13] J. S. Levinger, Phys. Rev. 87, 656 (1952).

[14] H. A. Bethe, (private communication in ref. 13).

[15] F. Rohrlich and N. Rosenzweig (private communication in ref. 13).

[16] G. Brown and J. Woodward, Proc. Phys. Soc. (London) [A] 65, 972 (1952).

[17] G. Brown et al., Proc. Royal Soc. 227-A, 51, 59 (1954).

[18] W. J. Freeman, Phys. Rev. 91, 1410 (1953).

[19] (a) M. F. Manning and L. Goldberg, Phys. Rev. 53, 662 (1938); (b) D. R. Hartree and W. Hartree, Proc Roy. Soc. (London) 149, 210 (1935).

[20] G. W. Brindley and P. Ridley, Proc. Phys. Soc. 50, 96 (1938).

[21] A. C. Aitken, Determinants and Matrices, 7th ed. (Oliver and Boyd Ltd., Edinburgh, 1952).

[22] G. W. Brindley and F. E. Hoare, Proc Roy. Soc., 159A, 395 (1937); Proc. Phys. Soc. 49, 619 (1937); Trans. Faraday Soc. 33, 268 (1937)

[23] D. R. Hartree and W. Hartree, Proc. Roy. Soc. (London) A15\%, 490 (1936).

[24] R. MeWeeny, Acta Cryst. 4, 513 (1951); 5, 463 (1952); 6, 631 (1953) and $\boldsymbol{\gamma}, 180$ (1954). W. Cochran, Acta Cryst. 6, 812 (1953).

[25] W. G. Henry, Acta Cryst. \%, 138 (1954).

[26] R. R. Wilson, Phys. Rev. 82, 295 (1951).

[27] A. K. Mann (private communication).

[28] A. Storruste, Proc. Phys. Soc. A53, 1197 (1950).

[29] W. E. Duncanson and C. A. Coulson, Proc. Roy. Soc. Edinburgh A62, 37 (1949).

[30] R. Brill, Acta Cryst. 3, 333 (1950).

[31] L. G. Parratt and C. F. Hempstead, Phys. Rev. 94, 1593 (1954).

[32] V. Schomaker and R. Glauber, Nature (Lond.) 170, 290 (1952); Phys. Rev. 89, 667 (1953).

[33] J. A. Ibers and J. A. Hoerni, Acta Cryst. 7, 405 (1954).

Washington, December 23, 1954. 